\title{
Contrats \\ et vivant : le droit \\ de la circulation \\ des ressources \\ biologiques \\ Florence Bellivier, \\ Christine Noiville
}

Hervé Chneiweiss

La réglementation, et donc le Droit, sont de plus en plus présents dans le quotidien des laboratoires. De plus, les avancées des connaissances en biologie et leur impact sur la société ont conduit à une législation de plus en plus précise et rigoureuse concernant le matériel biologique, tout particulièrement lorsqu'il s'agit de matériel humain. La patrimonialité des ressources biologiques, les règles du transfert et de la propriété intellectuelle, les règles sanitaires, nous obligent à comprendre, si ce n'est aussi apprendre, le Droit de la recherche en biologie. L'ouvrage que nous proposent aujourd'hui Florence Bellivier, professeure agrégée de droit, enseignante à I'Université Paris X Nanterre, et Christine Noiville, docteure en droit et chargée de recherche au CNRS est à plus d'un titre nécessaire et appelé à devenir une référence incontournable [1].

L'analyse des lois et règlements

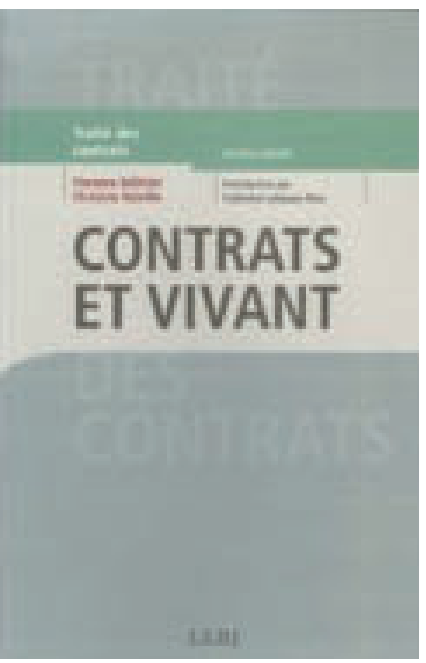

l'ouvrage se confronte par le biais de l'analyse des pratiques contractuelles. Il était devenu urgent, dans un contexte où des discours plus ou moins informés et cohérents prospèrent sur ces sujets, d'effectuer une analyse dépassionnée mais engagée de quelques 120 contrats choisis dans les trois règnes du vivant (végétal, animal, humain) et émanant tant d'institutions publiques de recherche que d'entreprises privées.

Dans la première partie, le livre aborde le contexte économique, technique et juridique des opérations portant sur le vivant. Le livre essaie de montrer comment le vivant est passé du statut d'objet de connaissance à celui d'objet d'exploitation et donc de débat sur le droit de la propriété et les règles de qui régissent les contrats sur le vivant permettent d'aborder des question d'une brûlante actualité éthique et économique, donc très politique. Ainsi au fil des chapitres, nous voyons apparaître l'import/export de cellules souches, la brevetabilité des gènes, les centres de ressources biologiques et les biobanques, l'accès aux soins, la recherche biomédicale telle qu'elle est organisée par les lois Huriet ou de bioéthique [2], la bioprospection dans les pays en voie de développement, le marché des organes : autant de domaines auxquels son commerce. Les auteurs ont fait ici le choix délibéré d'une lecture technique qui s'appuie sur l'idée de suivre des cas concrets: que fait le chercheur qui met de l'ADN dans une biobanque? Effectue-t-il un dépôt et quelles sont les conséquences juridiques que cela implique? Qu'est-ce que le «don bioéthique » par lequel un individu autorise le prélèvement de quelques cellules de son corps?

Dans la seconde partie, l'analyse est beaucoup plus technique et nous sommes convié à suivre pas 
à pas les contrats, leur source, leurs raisons, qui président à la collecte, au stockage, au transfert, à l'étude, etc..d'échantillons biologiques. D'intérêt également le chapitre qui aborde la question de la diffusion des connaissances, de plus en plus de contrats entre partenaires publics et privés restreignant ou imposant des délais à la diffusion des résultats de la recherche. Rien n'est unique et plusieurs modèles de contrats sont possibles. Intéressant pour votre prochaine négociation avec un industriel de la pharmacie!

Un tel angle d'attaque doit forcément être complété par une analyse politique de la contractualisation du vivant, tant l'articulation de l'intérêt général et des volontés privées est l'enjeu primordial de la recherche sur le vivant. C'est le thème développé par la troisième partie de l'ouvrage. Dans quelle mesure et sous quelle forme la loi doit-elle intervenir pour faire respecter la protection des donneurs [3-5] ou celle de l'environnement, pour assurer le partage équitable des bénéfices tirés de la recherche ? Une convention internationale est-elle souhaitable et possible pour élaborer le régime d'une exploitation du vivant qui serait à la fois juste et efficace?

À travers la palette des contrats et leurs conséquences, nous découvrons qu'il est inutile d'opposer contrat et brevet mais que les deux notions doivent évoluer en matière de vivant. Ainsi, Christine Noiville indiquait-elle déjà en 2005 dans une conférence organisée par la commission française auprès de I'UNESCO sur le partage des biotechnologies: «D'un côté, le brevet est une technique d'appropriation, de réservation au profit de l'inventeur, qui rime avec monopole, exclusivité, c'est-à-dire le contraire du partage - même si, en vérité, les choses sont plus subtiles puisque le brevet étant publié, l'invention est divulguée à tous et qu'une exemption de recherche permet de l'utiliser pour des besoins privés ou pour la tester -, une exclusivité très forte dans le domaine des biotechnologies, au point que dans de nombreux exemples, elle aboutit à la confiscation et au blocage. De l'autre côté, il est vrai que pour contrer cette poussée d'exclusivisme, les acteurs ont parfois recouru au contrat au sens large du terme (accord de volontés). Exemple: lorsque les participants au déchiffrage du génome humain, puis des génomes animaux et végétaux se sont entendus sur le principe de mise dans le domaine public des résultats issus de leurs travaux, c'est par contrat qu'ils ont convenu de prévenir tout monopole. C'est pourquoi le contrat a pu être considéré comme un outil rejetant le brevet. »

Rédigé dans un style accessible à tous les publics, l'ouvrage constitue un instrument indispensable aux différents acteurs impliqués dans le monde des biotechnologies (juristes, médecins, chercheurs, économistes ou sociologues de la santé, entrepreneurs, etc.) ou à tous ceux qui souhaitent s'initier à la matière. $\diamond$

Contracts and living: the law of the traffic

of the biological resources

\section{RÉFÉRENCES}

1. Bellivier F, Noiville C. Contrats et vivant : le droit de la circulation des ressources biologiques. Préface de Catherine Labrusse-Riou. Paris : LGDJ, $2006: 322 \mathrm{p}$.

2. Chneiweiss H. Chroniques bioéthiques (6). La révision des lois de bioéthique : une assemblée peut en cacher une autre. Med Sci (Paris) $2004 ; 20: 374-6$.

3. Chneiweiss H. De l'innovation en matière de médicaments à la thérapeutique : nouvelle donne éthique. Med Sci (Paris) 2004 ; $20: 259-60$.

4. Chneiweiss H. chroniques bioéthiques (5) Sur les rivages de la misère Épisode 1 : le marché des médicaments essentiels. Med Sci (Paris) 2003 ; $19: 892-4$.

5. Chneiweiss H. Chroniques bioéthiques (3). Dans les grandes plaines de la génomique. Épisode 1: le marché de la double hélice. Med Sci (Paris) $2003 ; 19: 500-4$

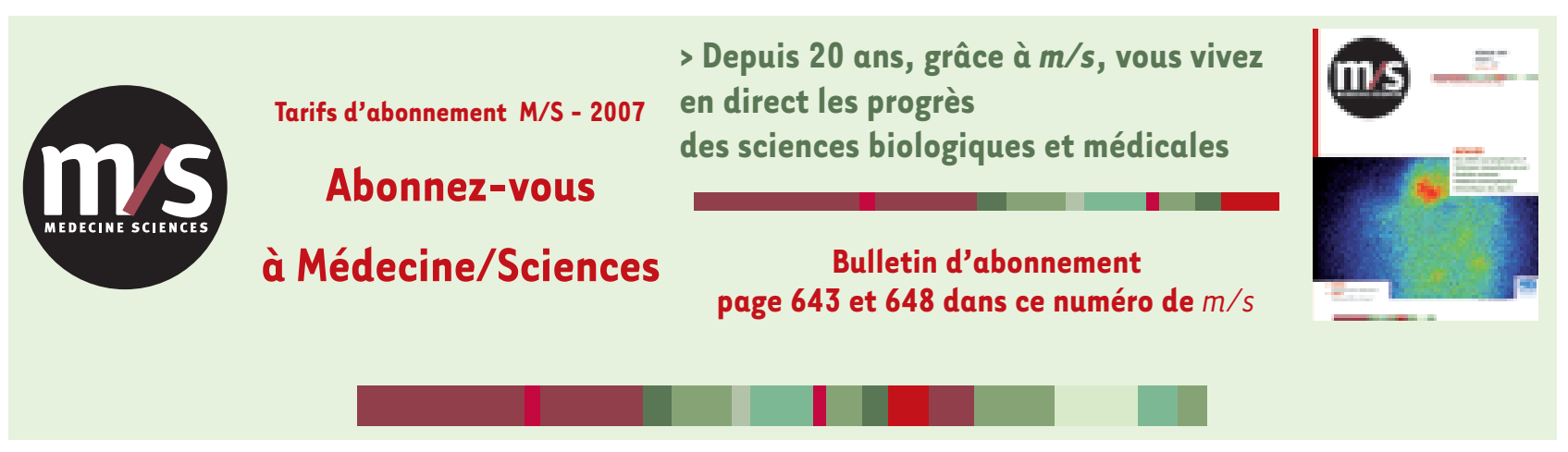

TIRÉS À PART

H. Chneiweiss 